ECCOMAS Proceedia
COMPDYN 2021

$8^{\text {th }}$ ECCOMAS Thematic Conference on Computational Methods in Structural Dynamics and Earthquake Engineering M. Papadrakakis, M. Fragiadakis (eds.)

\title{
MACHINE LEARNING TECHNIQUES FOR THE ESTIMATION OF LIMIT STATE THRESHOLDS AND BRIDGE-SPECIFIC FRAGILITY ANALYSIS OF R/C BRIDGES
}

\author{
Sotiria P. Stefanidou ${ }^{1}$, Vassilis K. Papanikolaou ${ }^{2}$, \\ Elias A. Paraskevopoulos ${ }^{1}$, Andreas J. Kappos ${ }^{3}$ \\ ${ }^{1}$ Postdoctoral Researcher, School of Civil Engineering, Aristotle University of Thessaloniki \\ Thessaloniki, 54124, Greece \\ e-mail: ssotiria@civil.auth.gr, eapcivil@gmal.com \\ ${ }^{2}$ Assistant Professor, School of Civil Engineering, Aristotle University of Thessaloniki \\ Thessaloniki, 54124, Greece \\ e-mail: billy@civil.auth.gr \\ ${ }^{3}$ Professor, Department of Civil Infrastructure and Environmental Engineering, Khalifa University \\ Abu Dhabi, United Arab Emirates; formerly, Professor, School of Civil Engineering, \\ Aristotle University of Thessaloniki, Thessaloniki, 54124, Greece \\ e-mail: andreas.kappos@ku.ac.ae
}

\begin{abstract}
Based on past earthquake events, bridges are the most critical and usually the most vulnerable components of road and rail transport systems, while bridge damage is related to substantial direct and indirect losses. In view of this, the need for direct and reliable assessment of bridge vulnerability has emerged, and several methodologies have been developed using probabilistic analysis for the derivation of fragility curves. A new framework for the derivation of bridge-specific fragility curves is proposed herein, introducing machine learning techniques for a reliable estimation of limit state thresholds of the most critical component of the bridge system (which in standard-ductility based-design is the piers), in terms of a widely used engineering demand parameter, i.e. displacement of control point. A set of parameters affecting the seismic capacity and the failure modes of bridge piers is selected, including geometry, material properties, and reinforcement ratios for cylindrical piers. Training and test sets are generated from multiple inelastic pushover analyses of the pier component, and Artificial Neural Networks (ANN) analysis is performed to derive closed-form relationships for the estimation of limit state thresholds. The latter are compared with closed-form relationships available in the literature, highlighting the effect of machine learning techniques on the reliable estimation of bridge fragility curves for all damage states.
\end{abstract}

Keywords: Bridge fragility curves, Limit state thresholds, Machine learning techniques, ANN 


\section{INTRODUCTION}

There is an increasing trend for assessment and retrofit prioritization of existing infrastructure in earthquake-prone areas, as well as investment planning, to be based on reliability analysis results. In this context, numerous methodologies have been proposed during the last 30 years for the derivation of bridge fragility curves, with increasing accuracy and efficiency over the years, introducing the use of advanced computational methods and probabilistic framework for the estimation of the probability of damage for different levels of earthquake intensity and limit states. The majority of the methodologies proposed are analytical, differing mainly in the quantitative definition of limit states (LS), i.e., the threshold values of LS considered, the type of analysis, the uncertainty treatment, and the probabilistic model used for the fragility analysis (probabilistic seismic demand model, response surface models, metamodels) $[1,2]$. Both generic and bridge-specific fragility curves have been proposed, accounting for the various uncertainties in seismic capacity and demand estimation of critical components, and a few of them for the effect of case-specific properties on the results.

Very recently [3], the potential exploitation of Artificial Intelligence algorithms and Machine Learning (ML) techniques in earthquake engineering, (i.e., seismic hazard analysis, system identification and damage detection, seismic fragility assessment, and structural control for earthquake mitigation) has been explored; it is still at a rather early stage, but a promising development. ML algorithms can be classified into supervised learning and unsupervised learning type. Supervised learning uses prior knowledge of the labeled data set to learn a function that best approximates the relationship between input and labeled output in the data. In contrast, unsupervised learning aims to infer the natural structure from a set of data points that have no target labels [3].

Several machine learning tools (i.e., classification-based tools like random forest, neural networks, etc.) have been proposed and applied to assess bridges and derive fragility curves. The efficiency of ML techniques for the derivation of fragility curves for different bridge classes and the identification of the effect of parameter uncertainties on the results have been studied for different bridge types and structural systems [4], [5]. Furthermore, the capabilities of machine learning techniques have been used to check the validity of assumptions during fragility analysis of bridges (e.g., the assumption of lognormal distribution of the demand model, etc.) [6]. Rapid damage assessment of bridges has been proposed based on analysis of selected bridge classes using the prediction model established via the training set and evaluating the performance of the model using the test set, as proposed in [5]. Based on the above, it is clear that the use of ML algorithms in the frame of fragility analysis of bridges is a new, promising approach that could eventually drastically reduce the computational effort and increase the accuracy and the efficiency of fragility analysis. However, since fragility analysis includes multiple steps and calculations (i.e., capacity, demand, uncertainty, etc.), the use of ML techniques at several stages should be properly addressed.

In view of the above, a methodology for the derivation of bridge-specific fragility curves that has recently been proposed by the authors [7], and is applicable to bridge stocks will serve as the starting point for setting up a ML procedure. The aim of this paper is to develop and apply ML algorithms (Artificial Neural Networks (ANN)) for a more reliable estimation of bridge pier capacity and limit state thresholds for all limit states (minor damage to collapse) considered in fragility analysis. A set of parameters affecting the seismic capacity and the failure modes of cylindrical bridge piers is initially selected, including geometry, material properties, axial load and reinforcement ratios. The range of parameters is selected with a view to representing realistic cases and resulting in training and tests of sufficient size. Analysis at local level (pier section) and global level (inelastic pushover analysis of bridge compo- 
nents) is performed and the results in terms of a widely used engineering demand parameter (EDP: displacement of control point/drift) are recorded. Test and training sets are generated from the results of numerous inelastic pushover analyses, and Artificial Neural Networks (ANN) analysis is performed, resulting in closed-form relationships for the estimation of limit state thresholds. The latter are compared with closed-form relationships derived through 'standard' regression analysis, in the frame of the methodology described in [7], to study the effect of ML techniques on the accuracy of the limit state threshold definition and, eventually, its potential for estimating bridge fragility curves for all limit states.

\section{COMPONENT-SPECIFIC LIMIT STATE THRESHOLDS AND PROPOSED CLOSED-FORM RELATIONSHIPS}

Utilising the methodology described in [7], component-specific limit state thresholds are estimated for bridge piers in terms of displacement at the component control point (pier top). Closed-form relationships relating $d / \mathrm{h}$ to the parameters affecting the seismic performance and ductility of bridge piers, namely geometry, material properties, longitudinal and transverse reinforcement ratios, and axial load, are derived for all limit states (minor damage to collapse). Limit state thresholds are initially defined qualitatively and subsequently quantitively at local (section) and global (component) level.

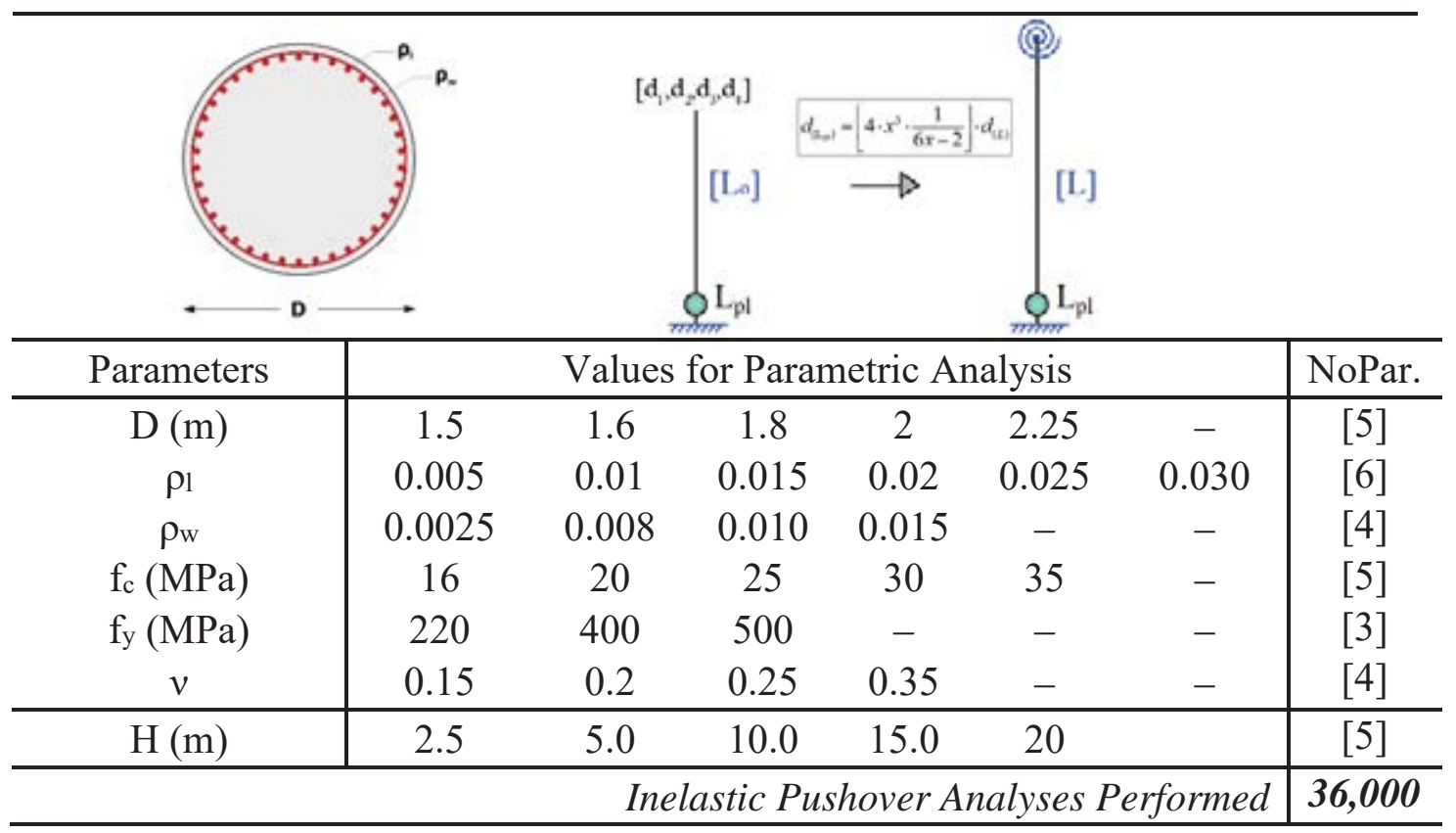

Table 1: Cylindrical pier section and component parameters considered.

A broad range of different pier section properties described in Table 1 are considered, and section analysis is performed using an in-house developed software that provides bilinearised moment-curvature $(\mathrm{M}-\varphi)$ curves [8]. Damage is initially quantified in terms of material strain limit values, namely $\varepsilon_{c}$ and $\varepsilon_{s}$ corresponding to experimentally observed crack widths, and moment corresponding to loss of bearing capacity for limit state 4 (post-peak $M=0.9 \cdot \mathrm{M}_{\max }$ ). More details regarding local limit state definition are available in [7]. Based on cross section analysis, moment-curvature curves are derived (and bilinearised) and curvature values corresponding to the aforementioned material strains are defined. Hence, damage is initially quan- 
tified in curvature terms (local EDPs $\varphi_{1}, \varphi_{2}, \varphi_{3}, \varphi_{4}$ ), and $M-\varphi$ curves, as well as effective stiffnesses EIeff ( $\mathrm{M}_{\mathrm{y}} / \varphi_{\mathrm{y}}$, needed for pushover analysis) are defined. Section analysis results for all possible parameter combinations (7,200 section analyses), are obtained; a sufficiently broad range of heights is considered, and parametric setup of an inelastic cantilever model (Fig.1) is performed in order to perform inelastic pushover component analysis (36,000 pushover analyses, see Table 1). Plastic hinge formation is considered at the pier base (lumped plasticity model), while the bilinear M- $\varphi$ curve is used as input. Inelastic pushover analysis is performed for all sections considered paired with all different pier heights, and the global engineering demand parameter values $\left(\mathrm{d}_{1}, \mathrm{~d}_{2}, \mathrm{~d}_{3}, \mathrm{~d}_{4}\right)$ are recorded at the analysis step that the relevant local values $\left(\varphi_{1}, \varphi_{2}, \varphi_{3}, \varphi_{4}\right)$ are exceeded. Check for shear failure, calculating the displacement $\left(d_{v}\right)$ when shear strength $V_{u}$ is exceeded is performed, considering reduced concrete contribution in the inelastic range [1]; $\mathrm{dv}$ is compared to the displacement at flexural failure $\left(\mathrm{d}_{\mathrm{fl}}\right)$ and the minimum value is considered as threshold value for the limit state (Fig.1).

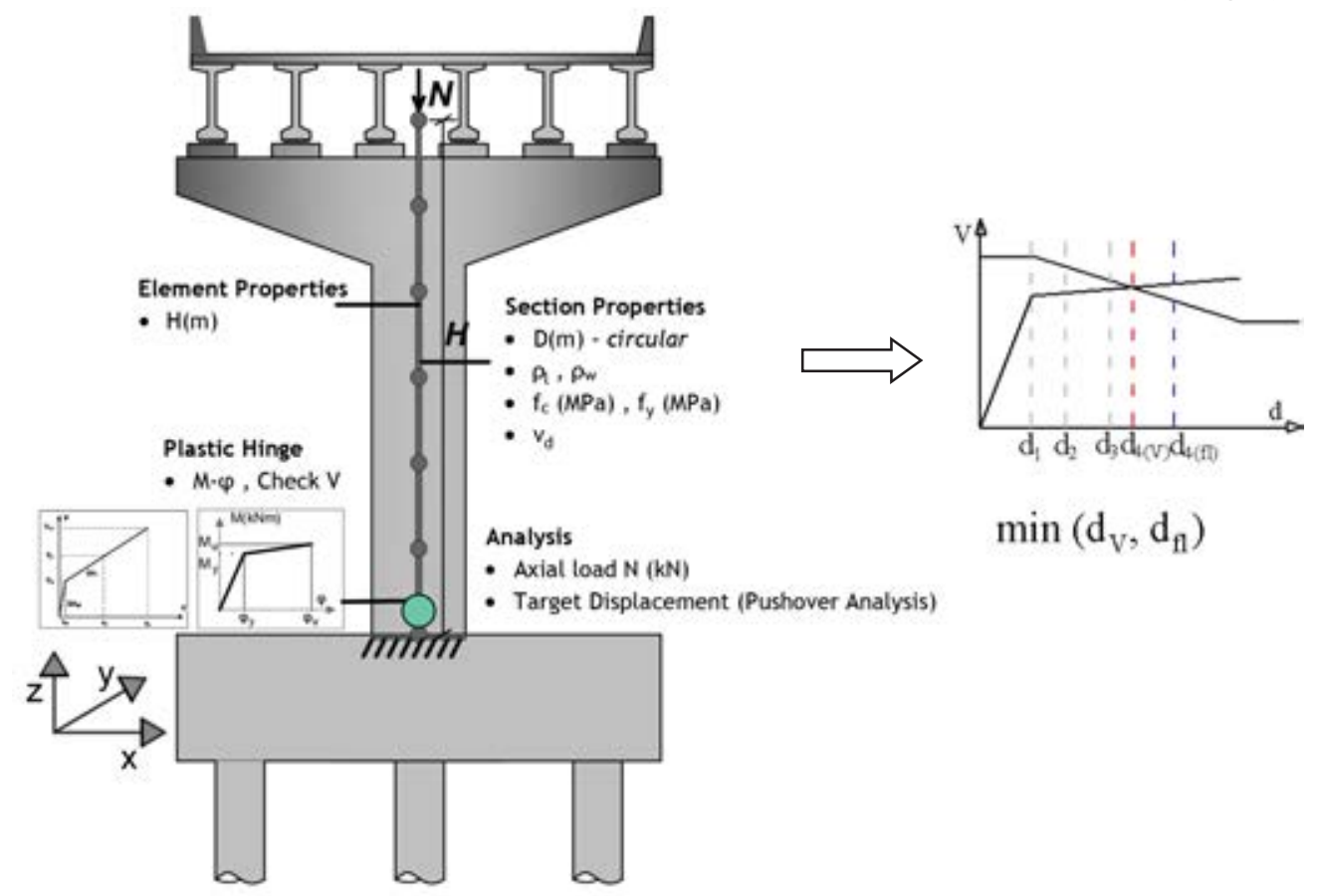

Figure 1: - Inelastic Pushover Analysis of the cantilever model and limit state threshold estimation

\begin{tabular}{c|c|c|c|c|c|c}
\hline \multicolumn{6}{c}{$\delta / \mathrm{H}=\exp \left[\beta_{0}+\beta_{1} \cdot \ln (\mathrm{D} / H)+\beta_{2} \cdot \ln (v)+\beta_{3} \cdot \ln \left(f_{c} / f_{y}\right)+\beta_{4} \cdot \ln \rho_{w}+\beta_{5} \cdot \ln \rho_{l}\right]$} \\
\hline & $\beta_{0}$ & $\beta_{1}$ & $\beta_{2}$ & $\beta_{3}$ & $\beta_{4}$ & $\beta_{5}$ \\
\hline$\delta_{1} / H$ & -6.524 & -0.876 & -0.018 & -0.688 & +0.086 & +0.292 \\
\hline$\delta_{2} / H$ & -6.016 & -0.674 & -0.265 & -0.076 & +0.030 & -0.072 \\
\hline$\delta_{3} / H$ & -3.872 & -0.572 & -0.238 & -0.470 & +0.505 & -0.108 \\
\hline$\delta_{4} / H$ & -3.663 & -0.542 & -0.381 & -0.518 & +0.439 & +0.001 \\
\hline
\end{tabular}

Table 2: Closed-form relationships based on regression of analysis results.

The pushover analysis results $\left(d_{1} / \mathrm{h} \sim d_{4} / \mathrm{h}\right)$ of the extensive parametric study are obtained, and regression analysis (considering the parameters in logarithmic form and performing nonlinear 
fit) is carried out to derive closed-form relationships for cylindrical piers. The proposed closed-form relationships for the estimation of limit state thresholds $\left(d_{1} \sim d_{4}\right)$ and, eventually, of seismic capacity, are provided in Table 2 .

\section{METHODOLOGY FOR THE DERIVATION OF LIMIT STATE THRESHOLDS USING ML TECHNIQUES (ANN)}

An alternative method for the estimation of pier LS threshold values in the context of bridge-specific fragility analysis is introduced herein, entailing the use of ML techniques, i.e.,Artificial Neural Networks (ANNs), trained to predict $\mathrm{d}_{1} \sim \mathrm{d}_{4}$ values calculated from inelastic pushover component analysis. The use of ANNs in engineering problems is common; however issues like high bias (i.e., not enough complexity to describe the input data) and high variance (i.e., overfitting), should be avoided, as described in [9], where additional information regarding ANNs is provided.

The first part of the methodology is the same as the one proposed in [7] and described in $\S 2$, i.e. a range of parameters is selected and paired in order to perform section and component analyses and estimate limit state thresholds in displacement terms. The range of parameters, selected in order to represent realistic cases and result in training and tests of sufficient size, is presented in Table 3.

\begin{tabular}{c|c|c|c}
\hline \multirow{2}{*}{ Parameters } & \multicolumn{2}{|c|}{ Training \& Test Sets } & Number of \\
\cline { 2 - 3 } & Range & Step & Param. \\
\hline $\mathrm{D}(\mathrm{m})$ & $1.5-2.3$ & 0.1 & {$[9]$} \\
$\rho_{1}$ & $0.005-0.03$ & 0.0025 & {$[11]$} \\
$\rho_{\mathrm{w}}$ & $0.0025-0.015$ & 0.0025 & {$[6]$} \\
$v$ & $0.1-0.3$ & 0.02 & {$[11]$} \\
\hline $\mathrm{f}_{\mathrm{c}}(\mathrm{MPa})$ & \multicolumn{2}{|c|}{$16,20,25,30$} & {$[4]$} \\
\hline $\mathrm{f}_{\mathrm{y}}(\mathrm{MPa})$ & \multicolumn{2}{|c|}{$220,400,500$} & {$[3]$} \\
\hline $\mathrm{H}(\mathrm{m})$ & \multicolumn{2}{|c|}{$2.5,5,10,15$} & {$[4]$} \\
\hline & \multicolumn{2}{|c|}{ Analyses Performed } & $\mathbf{3 1 3 , 6 3 2}$ \\
\hline
\end{tabular}

Table 3: Parameters considered for section and inelastic pushover analyses for training and test of ANNs

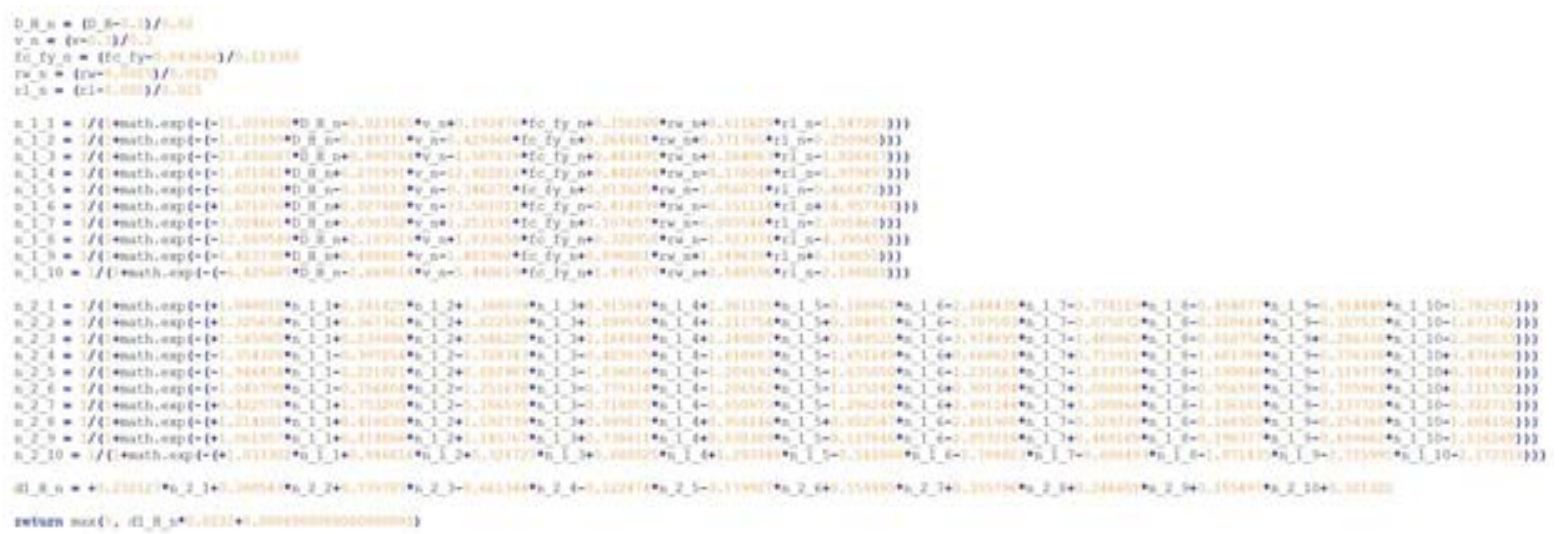

Figure 2: Function code (python) for $\mathbf{d}_{1}$ closed-form relationship 
A total of 313,632 pushover analyses were performed and used for training and testing of ANNs (half of the total set size used for training and the other half for testing). Different ANN sizes were examined (i.e. 5-5-5-1, 5-5-5-5, 5-10-10-1, 5-15-15-1) to select the most efficient in terms of recorded MAE (mean absolute error) and MPE/MNE (maximum positive and negative errors). The 5-10-10-1 (two inner layers of 10 nodes each) was found the most appropriate ANN size, since the recorded errors did not differ significantly from the ones of 5-1515-1 size and were apparently lower than those recorded when 5-5-5-5 was applied. The training procedure produced four closed-form relationships (functions) for the considered pier type (cylindrical pier) and all limit states. All analyses presented herein have been carried out using Python / TensorFlow 2.5 and a typical format of the relationship proposed for $d_{1}$ is provided in Figure 2. Testing of the relationships for $\mathrm{d}_{1} \sim \mathrm{d}_{4}$ was performed using the test set described above, estimating the errors to establish the accuracy of the proposed relationships and their efficiency towards limit state thresholds estimation.

\section{COMPARISON}

To evaluate and compare the closed-form relationships proposed herein based on the training/testing of ANNs, with those proposed in [7], derived from regression analysis, the diagrams of drift $(d / \mathrm{h})_{\text {analysis }}$ values and the error resulting from the proposed relationships $\left((d / \mathrm{h})_{\text {predicted- }}\right.$ $\left.(d / h)_{\text {analysis }}\right)$ are calculated for both cases, providing the relevant plots and the fit indicators, i.e. the MAE, MPE, MNE and the RMSE (Root Mean Squared Error) and R $^{2}$ (Root Mean Squared Error).

Closed-form relationships calculated from ANN

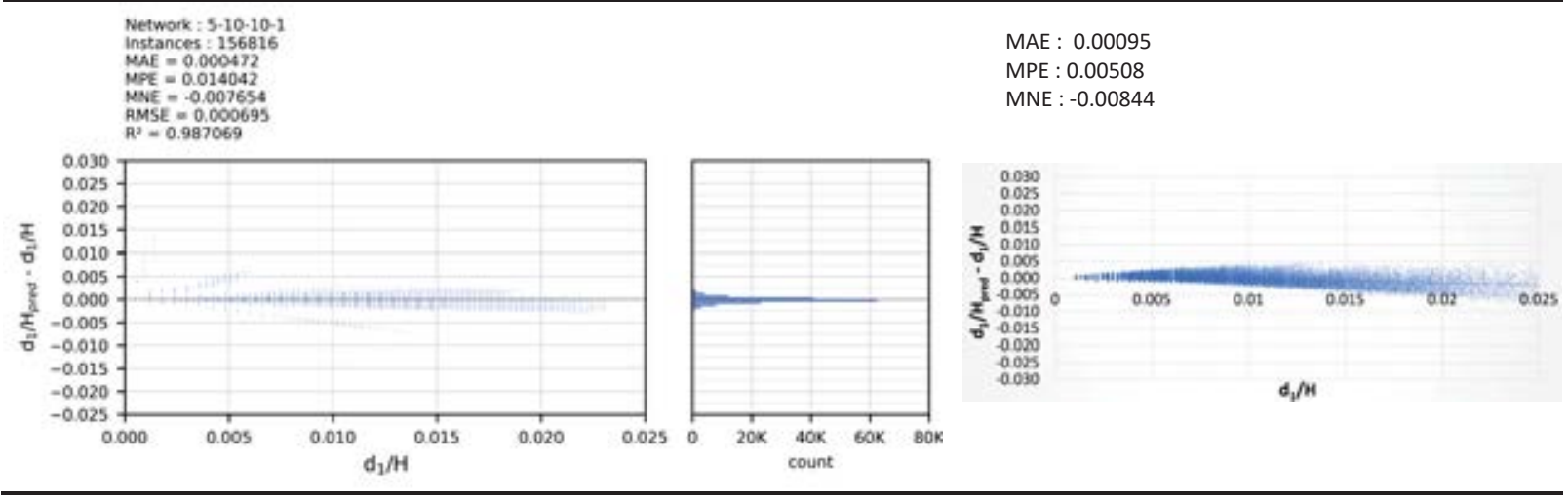

Figure 3: Comparison of $\mathbf{d}_{\mathbf{1}}$ prediction error of the closed-form relationships proposed in [7] and application of ML techniques (ANN)

Closed-form relationships calculated from ANN

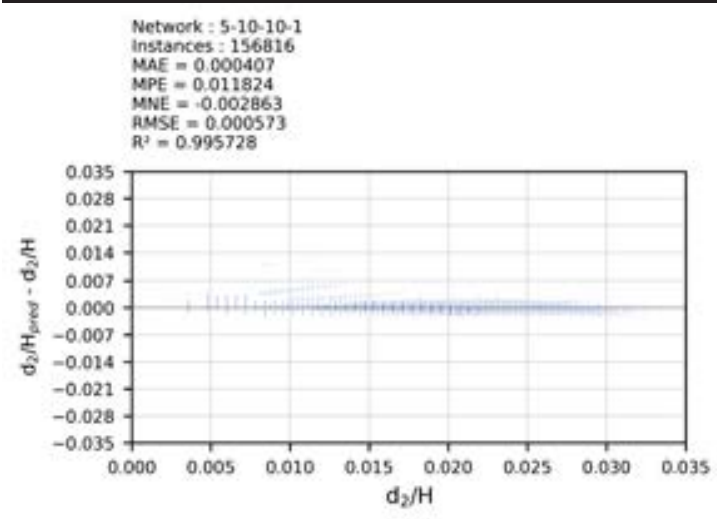

Closed-form relationships [7]

MAE : 0.00110

MPE : 0.00456 MNE : -0.00851

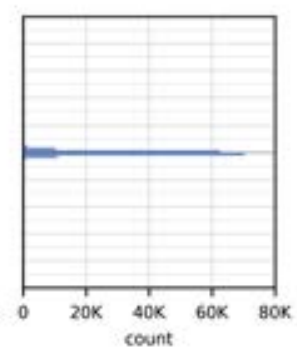

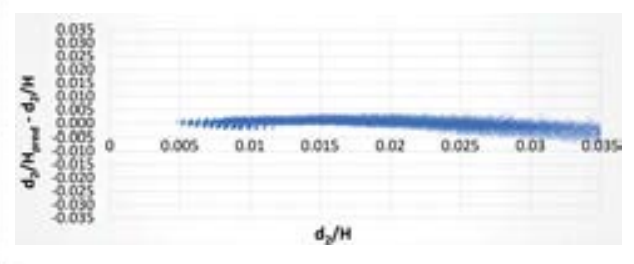


Figure 4: Comparison of $\mathbf{d}_{2}$ prediction error of the closed-form relationships proposed in [7] and application of ML techniques (ANN)

Closed-form relationships calculated from ANN Closed-form relationships [7]

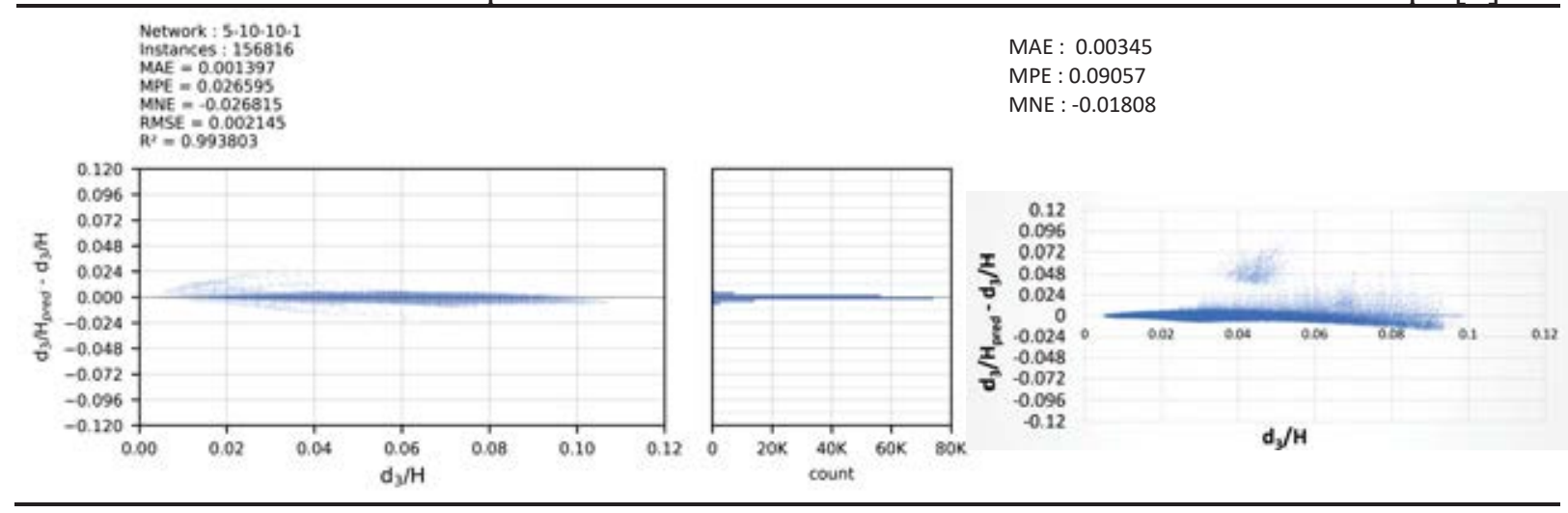

Figure 5: Comparison of $\mathbf{d}_{3}$ prediction error of the closed-form relationships proposed in [7] and application of ML techniques (ANN)

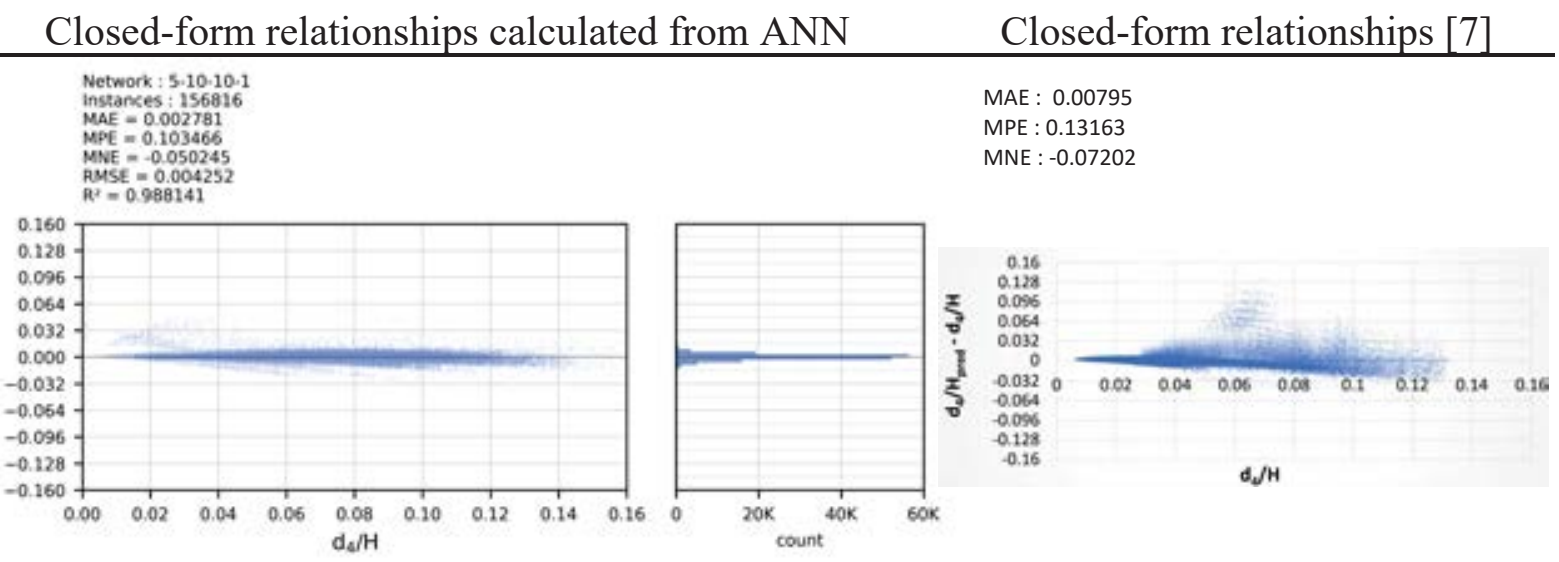

Figure 6: Comparison of $\mathbf{d}_{4}$ prediction error of the closed-form relationships proposed in [7] and application of ML techniques (ANN)

From Figures 3 to 6 it is clear that the values of MAE, MPE, MNE are lower in the case of the relationships proposed herein based on ANN training, compared to the ones proposed in [7]. The new relationships show a better fit of data, on the basis of the low RMSE and high $\mathrm{R}^{2}$ values (0.987 0.996 instead of $0.75 \sim 0.80$ of the new relationships), i.e. better prediction of the LS thresholds for all LS. The reduction of the prediction to analysis mean absolute error (MAE) ranges from 2 to 2.85 times, while it is obvious that the error reduction is larger for the higher limit states $\left(\mathrm{d}_{3} \& \mathrm{~d}_{4}\right)$, wherein the data fit based on regression analysis proposed in [7] was found inadequate.

\section{CONCLUSIONS}

The effectiveness of ML algorithms (ANN) for the quantitative estimation of limit state thresholds and the derivation of closed-form relationships (functions) used for seismic capacity estimation in the frame of a bridge-specific methodology proposed by the authors [7] was investigated herein. The closed-form relationships proposed within the methodology described in [7] which are based on regression of parametric inelastic analysis results of bridge piers are compared with those estimated based on extensive parametric analysis results used 
for training and testing of ANNs. The parameters considered for both cases are the same (geometry, material properties, axial load and reinforcement ratios), however the approach proposed herein is differentiated regarding the number of parametric analysis performed for the development of training and test sets and the application of ANNs, instead of typical regression analysis. The most important findings related to the effectiveness of ML techniques and the increased accuracy achieved during the quantitative limit state threshold definition and, eventually, the estimation of bridge fragility curves for all damage states, are:

- The relationships derived herein based on ANN training, have lower error (MAE, MPE, $\mathrm{MNE}$ ) and high $\mathrm{R}^{2}$ values, indicating better fit of data; i.e. better prediction of the LS thresholds for all LS, compared to those proposed in [7] derived from regression analysis.

- The reduction of the prediction to analysis mean absolute error (MAE) ranges from 50\% to $65 \%$ when ANNs are used for closed-form relationship estimation.

- The error reduction is larger for the higher limit states $\left(\mathrm{d}_{3} \& \mathrm{~d}_{4}\right)$, where the data fit based on regression analysis proposed in [7] was found inadequate. The latter is due to the increased sample size used and the training/testing of ANNs.

- The relationships proposed herein for the estimation of limit state thresholds for cylindrical piers $\left(\mathrm{d}_{1} \sim \mathrm{d}_{4}\right)$ indicate in general better fit to analysis data, hence providing more accurate and reliable prediction of component capacity and, eventually, seismic fragility.

\section{ACKNOWLEDGEMENTS}

"This research was co-financed by Greece and the European Union (European Social FundESF) through the Operational Programme «Human Resources Development, Education and Lifelong Learning 2014- 2020» in the context of the project "Online database for the development of fragility curves for as-built and retrofitted RC bridges using machine learning techniques" (MIS 5047878).

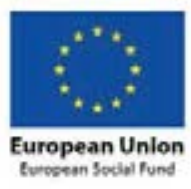

Operational Programme Human Resources Development, Education and Lifelong Learning

Co-financed by Greece and the European Union
EЕПA

2014-2020

\section{REFERENCES}

[1] S.P. Stefanidou, \& A. J. Kappos. Bridge Specific Fragility Analysis: When is it really necessary? Bulletin of Earthquake Engineering, 17(4), 2245-2280, 2019.

[2] S.P. Stefanidou \& A.J. Kappos. Fragility-informed selection of bridge retrofit scheme based on performance criteria. Engineering Structures, 234, 111976, 2021

[3] Y. Xie, M. Ebad Sichani, J. E. Padgett, \& R. DesRoches. The promise of implementing machine learning in earthquake engineering: A state-of-the-art review. Earthquake Spectra, 36(4), 1769-1801, 2020 
[4] S. Mangalathu, G. Heo, \& J.S. Jeon. Artificial neural network based multi-dimensional fragility development of skewed concrete bridge classes. Engineering Structures, 162, 166-176, 2018.

[5] S. Mangalathu, S.H. Hwang, E. Choi \& J.S. Jeon. Rapid seismic damage evaluation of bridge portfolios using machine learning techniques. Engineering Structures, 201, $109785,2019$.

[6] S. Mangalathu \& J.S. Jeon. Stripe-based fragility analysis of multispan concrete bridge classes using machine learning techniques. Earthquake Engineering and Structural Dynamics, 48(11), 1238-1255, 2019.

[7] S.P. Stefanidou \& A.J. Kappos. Methodology for the development of bridge-specific fragility curves. Earthquake Engineering \& Structural Dynamics, 46, 73-93, 2017.

[8] V.K. Papanikolaou, Analysis of arbitrary composite sections in biaxial bending and axial load. Computers \& Structures, 98-99, 33-54, 2012.

[9] A.E. Charalampakis \& V.K. Papanikolaou. Machine learning design of R/C columns. Engineering Structures, 226, 111412, 2021. 